\title{
Ideologías curriculares en el debate y negociación del currículum chileno de Historia, Geografía y Ciencias Sociales*
}

\author{
Curricular ideologies in the discussion and negotiation of the \\ Chilean curriculum of Social Studies
}

\author{
Renato Gazmuri Stein \\ Doctor en Didáctica de la Historia, la Geografía y las Ciencias Sociales, Universidad Autónoma de Barcelona \\ Telf.: (56) 226768501. Correo electrónico: renato.gazmuri@udp.cl
}

\section{RESUMEN}

El currículum chileno de Historia, Geografía y Ciencias Sociales se ha definido a través de procesos de debate y negociación entre una diversidad de actores e instituciones con distintas visiones sobre esta asignatura. En esta investigación se identifican y describen las principales ideologías curriculares que sostienen estos actores y agencias. Se utilizó un dispositivo metodológico secuencial y recursivo que consideró tres etapas de producción y análisis de información: una recopilación documental en torno a tres eventos de debate y negociación curricular, la aplicación de cuestionarios y la realización de entrevistas. En cada etapa se realizó un análisis de contenido. Se identificaron cinco ideologías curriculares, las que se describen considerando sus postulados sobre la forma en que se debe definir el currículum de la asignatura, cuáles deben ser sus finalidades, sus contenidos y sus orientaciones para la enseñanza.

Palabras clave: currículum, historia, sociedad, ciudadanía, esquema de ideologías.

\section{ABSTRACT}

The Chilean curriculum of Social Studies has been defined through processes of discussion and negotiation between diverse actors and institutions with different views on the subject. In order to identify and describe these ideologies, a sequential and recursive methodological device was designed and applied in three stages of production and analysis of information: a documentary compilation around three curricular events of debate and negotiation, application of questionnaires, and interviews. At each stage a content analysis was performed. Five curriculum ideologies are identified and described, considering their assumptions about how the curriculum should define the subject, as well what its aims, contents and its guidelines for teaching.

Key words: curriculum, history, society, citizenship, ideologies schema.

\footnotetext{
Esta investigación se desarrolló en el marco de los estudios doctorales del autor, financiados por la Comisión Nacional de
} Investigación Científica y Tecnológica (CONICYT), a través del programa Becas Chile Doctorado. 


\section{INTRODUCCIÓN}

En las tres últimas versiones del currículum chileno (1996-1998, 2009 y 2012-2013), los contenidos sobre la historia, la sociedad y la ciudadanía se han organizado en asignaturas interdisciplinarias cuyo propósito fue que los estudiantes desarrollaran los conocimientos, habilidades y actitudes que les permitiesen una comprensión crítica de la sociedad y una valoración de la democracia y los derechos humanos. En los Marcos Curriculares de 19961998 la asignatura se denominaba "Estudios de la Sociedad" desde 5 a $8^{\circ}$ básico, e "Historia y Ciencias Sociales" en la Educación Media (Mineduc, 1996, 1998). En el Ajuste Curricular del 2009 y en las Bases Curriculares 2012-2013, en cambio, se definió una asignatura común para la enseñanza básica y media, con el nombre de "Historia, Geografía y Ciencias Sociales" (Mineduc, 2009, 2012, 2013).

Estas organizaciones curriculares y estos propósitos educativos son coherentes con las propuestas contemporáneas de la Didáctica de la Historia y las Ciencias Sociales, así como con la tendencia curricular en las democracias occidentales. En ambos referentes se promueve el estudio integrado de las ciencias sociales y las humanidades para el desarrollo de una conciencia ciudadana comprometida con la democracia y el respeto y ejercicio de los derechos humanos (Barton \& Levstik, 2004; Benejam, 1997; Carretero, 2007; Gazmuri, 2010; National Council for the Social Studies, 2010; Pagès, 2002).

En las últimas dos décadas, estas asignaturas no solo han orientado y regulado la enseñanza de la historia y las ciencias sociales en el país, sino que, además, han definido las nociones de historia, cultura, territorio, sociedad, democracia y ciudadanía con las que el Estado se ha comprometido para ser transmitidas, a través del sistema escolar, a las siguientes generaciones. Dada esta importancia práctica y simbólica que poseen las prescripciones curriculares (Goodson, 2000), los procesos a través de los cuales se definieron estas asignaturas y sus contenidos, convocaron el interés y la participación de una diversidad de actores e instituciones, no solo vinculados con la enseñanza escolar de la historia y las ciencias sociales (profesores, formadores de profesores, didactas y curriculistas), sino también de actores e instituciones provenientes del mundo político y de las disciplinas de referencia.

Entre esta diversidad de actores e instituciones, situados en distintos campos de la sociedad, existen también diversas concepciones respecto a la historia, la cultura, el territorio, la sociedad, la democracia y la ciudadanía, así como sobre el conocimiento, la enseñanza y el aprendizaje. Resulta de interés, entonces, identificar y describir las principales ideologías curriculares (Schiro, 2007) en Historia y Ciencias Sociales que sostienen estos actores e instituciones, con el objetivo de lograr una visión esquemática de los principales discursos que promueven quienes han participado del debate y negociación del currículum de esta asignatura en la historia reciente.

\section{MARCO TEÓRICO}

Tanto la teoría curricular como la sociología de la educación y la pedagogía crítica coinciden al señalar que las asignaturas escolares no son definiciones científicas ni neutras, sino más bien construcciones históricas y sociales que se desarrollan a través de procesos de debate y negociación entre una serie de actores e instituciones provenientes desde dentro y desde fuera del campo educativo (Apple, 1996; Bernstein, 1993; Goodson, 2003; Gvitz \& Palimedessi, 
2005; Popkewitz, 1998). En ese sentido, Igor Goodson plantea que la definición de una asignatura, de sus finalidades y contenidos "no consiste en una decisión imparcial, racional, sobre lo que se juzga de interés para los alumnos", sino más bien un acto político en el que participan distintos grupos de interés (Goodson, 2000, p. 43). De manera similar, Basil Bernstein (1993) concibe al currículum como un dispositivo pedagógico que se desarrolla al interior de un espacio de posiciones, en el que intervienen actores y agencias provenientes de distintos ámbitos o campos: desde el campo de la producción, desde el campo de la política o desde los campos intelectuales y pedagógicos. En este espacio, estos actores y agencias disputan y definen el qué y el cómo del discurso pedagógico que trasmite el currículum.

En las democracias contemporáneas en las que existe un currículum común o nacional, como en el caso chileno, este proceso de conflictos y compromisos entre distintos grupos se desarrolla en un escenario de negociación que se produce al alero del Estado. En este proceso, aquellos grupos que detentan más poder y pueden ejercer mayor presión se verían constreñidos a incorporar, a su vez, otros intereses de quienes detentan menor poder económico, cultural o político, en orden a dotar de legitimidad al conocimiento seleccionado. Así, se construye un consenso que actúa como una suerte de "paraguas" que protege al mayor número posible de grupos (Apple, 1996).

Esta diversidad de actores e instituciones que participan del proceso de debate y negociación curricular describen, a su vez, distintas creencias, intereses y visiones de mundo, así como distintas concepciones sobre el currículum y las asignaturas. Según Michael Schiro (2007), estas visiones y concepciones pueden ser concebidas como ideologías curriculares, entendidas como esquemas de pensamiento sobre el conocimiento, la enseñanza y el aprendizaje, que permean la visión de los sujetos sobre el currículum, así como sobre sus finalidades, contenidos y orientaciones para la enseñanza.

Si bien en la investigación educativa existen estudios sobre ideologías curriculares, estos se han abocado a identificar y describir las principales ideologías respecto al currículum común en distintos contextos nacionales y temporales (Joseph et al., 2000; Kliebard, 2004; Schiro, 2007; Schubert, 1996). No existen antecedentes de investigaciones equivalentes sobre ideologías curriculares respecto a las asignaturas o áreas curriculares relativas a la historia, la sociedad y la ciudadanía.

Por su parte, en la investigación sobre la enseñanza de la Historia y las Ciencias Sociales existen trabajos que profundizan en modelos o tradiciones de enseñanza (Benejam, 1997; Páges, 1998), pero no hay antecedentes de investigaciones sobre las ideologías curriculares que sostienen quienes debaten y definen el currículum de la asignatura. En otras palabras, se ha explorado la pregunta en torno a las distintas maneras de concebir y practicar su enseñanza, no así sobre las visiones con respecto a la asignatura que poseen quienes participan de su definición.

Interesa, entonces, identificar y describir las principales ideologías curriculares que efectivamente participan del proceso de debate y negociación del currículum chileno de Historia, Geografía y Ciencias Sociales, y vincularlas a los actores e instituciones que las sostienen.

\section{METODOLOGÍA}

Para identificar y describir las principales ideologías curriculares que han participado en los procesos de debate y negociación del currículum de Historia y Ciencias Sociales en la 
Estudios Pedagógicos XLIII, N $^{\circ}$ : 157-169, 2017

IDEOLOGÍAS CURRICULARES EN EL DEBATE Y NEGOCIACIÓN DEL CURRÍCULUM CHILENO DE HISTORIA, GEOGRAFÍA Y CIENCIAS SOCIALES

historia reciente, se diseñó un dispositivo metodológico de carácter recursivo e inductivo (Strauss \& Corbin, 2002). Este dispositivo se desarrolló en tres etapas. En cada una de ellas se utilizaron distintas técnicas de producción de información: una recopilación documental en torno a tres eventos (Popuart, 1997) en los que se debatió el currículum de Historia y Ciencias Sociales, la aplicación de cuestionarios de preguntas abiertas y entrevistas semiestructuradas a una selección de actores que participaron de estos eventos de debate y negociación curricular.

Para analizar la información producida se realizó un análisis de contenido, en el que se organizó la información en un conjunto de categorías apriorísticas e inductivas (Elliot, 1990), con el propósito de "extraer cierta estructura a partir del material” (Mayring, 2014, p. 95) en la forma de un sistema de categorías. Este análisis se desarrolló de manera recursiva en cada una de las etapas del dispositivo metodológico, en búsqueda de la saturación teórica de las categorías (Carrero, Soriano \& Trinidad, 2012).

\subsection{ETAPA 1. LA RECOPILACIÓN DOCUMENTAL Y SU ANÁLISIS}

Para la recopilación documental se definió una muestra por eventos. Es decir, una muestra teórica en la que se seleccionan eventos como "punto de anclaje empírico", los que "por regla general, son relativamente raros desde el punto de vista de la frecuencia" [eventos] "que se producen con el concurso de las instituciones y la cultura y que, por esta razón, permiten acceder a esta última en acción” (Popuart, 1997, pp. 136-137).

Se seleccionaron tres eventos: La Comisión Formación Ciudadana (2004), el Ajuste Curricular (2009) y el debate producido en torno al Decreto Ministerial que disminuía las horas de Historia, Geografía y Ciencias Sociales (2010). Para la selección de los eventos se aplicaron tres criterios: eventos de debate y negociación curricular de amplia convocatoria, eventos con consecuencias en el currículum prescrito y eventos con registros de los actores e instituciones participantes, así como de sus propuestas y postulados.

La Comisión Formación Ciudadana del 2004 fue un comité asesor convocado por el Ministerio de Educación con el objetivo de "examinar los requerimientos formativos que plantea la ciudadanía democrática" (Mineduc, 2004, p. 10). En ella participaron 17 miembros, 4 representantes del Ministerio de Educación y 17 expositores. Si bien la Comisión tuvo un objetivo amplio, en su informe final se manifestó una especial preocupación por la dimensión curricular de la formación ciudadana, y entre sus recomendaciones destacan la necesidad de incorporar una serie de contenidos en el currículum de Historia, Geografía y Ciencias Sociales. En este evento se analizó la documentación correspondiente a las actas de cada una de sus 11 sesiones, lo que se tradujo en 32 registros textuales. Esto es, dichos o escritos de un actor o agencia que expresan consideraciones o argumentos relativos a distintas dimensiones del currículum nacional de Historia, Geografía y Ciencias Sociales.

El segundo evento es el Ajuste Curricular 2009, una reforma a los marcos curriculares de enseñanza básica y media desarrollado por el Ministerio de Educación que consideró un proceso de consulta a un conjunto de organismos públicos, instituciones académicas y especialistas pedagógicos y disciplinarios. En este evento se analizaron 29 registros textuales, correspondientes a los informes elaborados por los actores e instituciones que participaron de este proceso.

Finalmente, el debate producido en torno al Decreto Ministerial del 2010 corresponde al debate público suscitado a propósito de un decreto anunciado por el Ministerio de 
Educación, el que disminuía de cuatro a tres las horas pedagógicas semanales destinadas a la asignatura de Historia, Geografía y Ciencias Sociales en los últimos siete años de la escolaridad. En este evento se analizaron 30 registros textuales, correspondientes a editoriales, columnas de opinión y cartas públicas publicadas en diferentes medios de comunicación.

Tabla 1. Eventos de negociación curricular, recopilación documental y registros

\begin{tabular}{|l|l|c|}
\hline Evento & Documentos & Registros textuales \\
\hline $\begin{array}{l}\text { Comisión Formación Ciuda- } \\
\text { dana 2004 }\end{array}$ & Actas e Informe Final & 32 \\
\hline Ajuste Curricular 2009 & $\begin{array}{l}\text { Consulta Pública } \\
\text { (informes y comentarios) }\end{array}$ & 29 \\
\hline $\begin{array}{l}\text { Debate Decreto Ministerial } \\
\text { disminución horas destina- } \\
\text { das a la asignatura, 2010 }\end{array}$ & $\begin{array}{l}\text { Editoriales, columnas de } \\
\text { opinión y cartas abiertas }\end{array}$ & 30 \\
\hline
\end{tabular}

Para el análisis de contenido, cada registro textual se clasificó de acuerdo a un conjunto de categorías previamente establecidas: actor o agencia que emitió los postulados, la condición en la que se expresa (como historiador, como autoridad política, como colectivo de profesores, etc.) y el evento de negociación curricular en el cual se pronunció.

Luego, cada registro se clasificó de acuerdo a un conjunto de categorías inductivas que respondían a las distintas dimensiones del currículum de Historia y Ciencias Sociales aludidas en los registros: la forma legítima de definir el currículum de la asignatura, cuáles deberían ser sus finalidades, los criterios para la selección y organización de los contenidos y sus orientaciones para la enseñanza.

Tras esta primera etapa se elaboró un primer borrador que organizaba, en un esquema, las ideologías curriculares en Historia y Ciencias Sociales que emergían del primer análisis del material.

\subsection{ETAPA 2. LA APLICACIÓN Y EL ANÁLISIS DE LOS CUESTIONARIOS}

En búsqueda de la saturación teórica de las categorías emergentes, se diseñó un cuestionario de preguntas abiertas con el objetivo de producir información sobre las categorías inductivas que emergieron del análisis documental. Este cuestionario se aplicó a una selección de 15 actores, que cumplían con la condición de haber participado en al menos uno de los tres eventos de negociación curricular. Para la validación del instrumento se recurrió al comentario experto de cuatro académicos especializados en teoría curricular, en didáctica de las ciencias sociales y en investigación educativa, además de la aplicación de un pilotaje del instrumento a cuatro profesionales vinculados a la enseñanza de la Historia y las Ciencias Sociales. Para el análisis de contenidos de la información producida a través de la aplicación de los cuestionarios se utilizaron las mismas categorías que para el análisis de los eventos. 


\subsection{ETAPA 3. LAS ENTREVISTAS Y SU ANÁLISIS}

Finalmente, se aplicó una entrevista semi-estructurada a cinco actores considerados como paradigmáticos de las distintas ideologías curriculares previamente identificadas y que habían participado de al menos uno de los eventos de negociación curricular. La pauta de este instrumento se diseñó con el objetivo de profundizar en las posiciones y argumentos de los actores seleccionados para, de esa manera, contar con información que permitiera saturar teóricamente las categorías y así precisar la descripción de las distintas ideologías curriculares. Las entrevista fueron transcritas y luego analizadas utilizando las mismas categorías que las etapas anteriores.

\section{RESULTADOS}

Se identificaron cinco ideologías curriculares. Cada una de ellas permite agrupar con coherencia los postulados y argumentos de un conjunto de actores e instituciones $\mathrm{y}$, al mismo tiempo, describen rasgos característicos que permiten distinguir entre unas y otras. Así, las ideologías identificadas fueron las siguientes: una ideología neoliberal, una ideología conservadora, una ideología liberal y dos ideologías críticas: una crítico-disciplinar y otra crítico-pedagógica.

Estas ideologías curriculares se presentan siguiendo un orden, desde aquellas más estrechamente ligadas con la transmisión o reproducción cultural, hacia aquellas más estrechamente vinculadas a la transformación cultural (Levstik, 1996). Al describir cada ideología se justifica su denominación, se identifican los campos de procedencia de los actores e instituciones que las sostienen, para luego exponer sus postulados sobre la forma en que se debería definir el currículum de esta asignatura, sus finalidades, los contenidos que este debiese considerar y sus orientaciones para la enseñanza.

Esquema 1. Ideologías curriculares en Historia, Geografía y Ciencias Sociales en relación a los polos de transmisión y transformación cultural

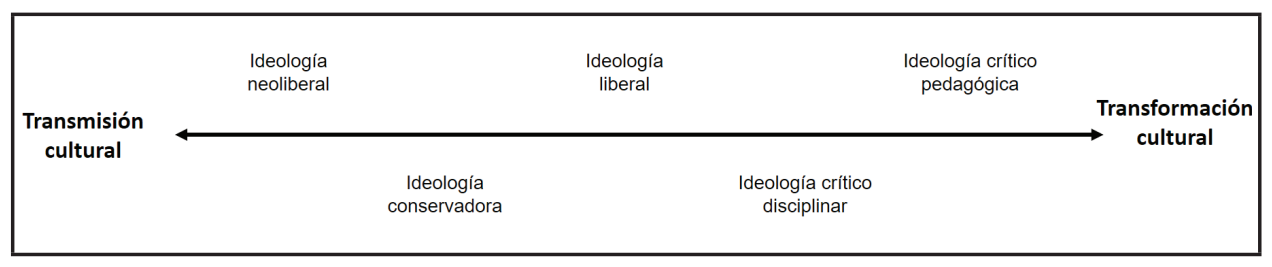

\subsection{LA IDEOLOGÍA NEOLIBERAL}

La denominación de esta ideología responde a que sus postulados son paradigmáticos del neoliberalismo, entendido como aquella visión del mundo según la cual el desarrollo y el progreso se logran a través de la libertad de empresa y la mínima injerencia del Estado.

Los actores e instituciones que se agrupan en esta ideología provienen de sectores del campo político, fundamentalmente de centros de pensamiento vinculados a partidos 
políticos, y del campo intelectual, desde las disciplinas de la economía y el derecho. Según estos actores e instituciones, el currículum debe ser elaborado por el Estado y sancionado por un organismo de control conformado por expertos.

El principal interés de esta ideología está puesto en la Educación Cívica y en la Economía. Su posicionamiento como una ideología abocada a la transmisión cultural responde, fundamentalmente, a que sus postulados buscan la valoración del orden económico y político vigente en Chile. Así, las finalidades de la Educación Cívica y la Economía en el currículum son la formación de un ciudadano respetuoso de las autoridades y las instituciones, además de comprometido con el crecimiento del país. En ese sentido, estas asignaturas deberían contribuir a que los estudiantes adscriban a valores vinculados a la realización individual y al emprendimiento.

Respecto a la Educación Cívica, esta debiese promover una concepción de la democracia entendida como un mecanismo de elección de autoridades y, en cuanto a los derechos, limitada a los derechos políticos y civiles. Respecto a la Economía, postula que debe tratarse como una ciencia exacta y, por tanto, debe considerar elementos objetivos como, por ejemplo, las leyes del mercado, así como nociones básicas de costos e innovación.

Para esta ideología, la enseñanza de estas asignaturas debe ser objetiva y evitar los juicios de valor.

\subsection{LA IDEOLOGÍA CONSERVADORA}

La denominación de esta ideología responde a que promueve la valoración de la tradición y el sentimiento de pertenencia a la nación.

Los actores e instituciones que sostienen esta ideología provienen del campo de la política y del campo intelectual. Se trata de políticos vinculados a los partidos de derecha, además de historiadores y juristas ligados al pensamiento conservador. Para estos actores e instituciones, el currículum de esta área debe responder a un consenso que considere solo aquellos contenidos sobre los que existe un acuerdo general y evitar los temas polémicos o contingentes.

Según la ideología conservadora, las finalidades fundamentales de la Historia, la Geografía y las Ciencias Sociales son el fortalecimiento de la identidad nacional y la formación de un ciudadano respetuoso de las instituciones y las leyes. Para estos objetivos, las disciplinas involucradas deberían organizarse separadamente, es decir, idealmente debería existir una asignatura de Historia, otra de Geografía y otra de Educación Cívica y Economía.

La Historia y la Geografía deberían organizarse en ciclos concéntricos en los que se replicaran las mismas temáticas en enseñanza básica y media, pero con distintos niveles de profundidad. Su selección temática debería considerar la historia universal y la tradición cristiano-occidental, así como la historia nacional, centrada en la evolución político, militar e intelectual de la nación, así como en los grandes personajes, en tanto modelos de virtud y patriotismo.

Por su parte, la asignatura de Educación Cívica y Economía debería estudiar la organización del sistema político y el funcionamiento del sistema económico, además de promover una concepción de la democracia como un proceso para la elección de autoridades. Junto con ello, debe considerar los derechos políticos y civiles, los deberes del ciudadano, la institucionalidad política nacional y la función de las autoridades. 
Para los actores e instituciones que sostienen la ideología conservadora, la enseñanza de estas asignaturas debe ser objetiva y evitar la contingencia y el conflicto.

\subsection{LA IDEOLOGÍA LIBERAL}

La denominación de esta ideología responde a que sus postulados se vinculan con el liberalismo político, entendido como una corriente de pensamiento que promueve las libertades civiles y la democracia representativa.

La ideología liberal describe un posicionamiento mixto en relación a los polos de transmisión y transformación cultural, en tanto pone énfasis en la valoración de cierta construcción nacional frente a la cual los estudiantes deberían sentir orgullo y lealtad, pero al mismo tiempo considera espacios para la crítica y la reflexividad.

Los actores e instituciones que detentan esta ideología provienen del campo de la política (políticos ligados a la socialdemocracia), del campo intelectual (instituciones y académicos vinculados al derecho, a la sociología y la historiografía) y del campo pedagógico (autoridades y funcionarios ligados a la política pública educativa, así como académicos vinculados a la educación y la formación de profesores).

Para estos actores, el currículum de la asignatura debería definirse a través de consensos políticos y pedagógicos de largo plazo, en orden a darle legitimidad y estabilidad. El currículum debiese ser propuesto por el Estado y consultado con actores políticos, especialistas disciplinarios, entidades formadoras de docentes y organismos de profesores.

Según esta ideología, las principales finalidades del área de Historia, Geografía y Ciencias Sociales deben ser la formación de un ciudadano racional y tolerante, capaz de comprender el presente y gestionar la incertidumbre, y la contribución a la cohesión social, a través de la generación de pertenencia a la comunidad cívica.

En ese sentido, se concibe a la formación ciudadana como un objetivo transversal al currículum y se promueve un sector curricular de Historia, Geografía y Ciencias Sociales que se desarrolle a lo largo de la escolaridad y que considere aportes de las distintas disciplinas en forma integrada. En ese marco, se le asigna un papel prioritario a la historia, la que debe dar cuenta de los relatos fundamentales de la nación, pero también al derecho, la sociología y la economía, en tanto se trata de disciplinas que entregan herramientas que permiten a los estudiantes alcanzar una comprensión de la sociedad en la que viven. Esta asignatura debiese promover una concepción de la democracia como un orden político legítimo y como un mecanismo para la resolución pacífica de conflictos, y contemplar los derechos políticos, civiles, económicos y sociales.

Según esta ideología, la enseñanza de la asignatura debe considerar una multiplicidad de métodos, como las clases lectivas y la organización de debates, pero también la simulación de conflictos, la producción de ensayos y la aproximación de los estudiantes a los métodos y objetos de estudio de las disciplinas de referencia.

\subsection{LAS IDEOLOGÍAS CRÍTICAS}

Las dos ideologías que se exponen a continuación reciben la denominación de críticas por la centralidad que tiene dentro de sus postulados la educación de ciudadanos críticos, comprometidos con la democratización de la sociedad. 
Los actores y agencias vinculados a estas ideologías provienen mayoritariamente del campo intelectual (académicos provenientes desde las disciplinas de referencia, sobre todo de la historia y, secundariamente, de la geografía) y del campo pedagógico (académicos provenientes de la formación de profesores, didactas del área y organizaciones docentes).

Para estos actores y agencias, el currículum nacional debería ser definido a través de un debate amplio que considere a los docentes, los estudiantes y a sus organizaciones, a académicos e investigadores del área de la educación y de las disciplinas de referencia, así como a todos aquellos grupos representados en el mensaje curricular.

Ambas ideologías comparten una similar concepción sobre cuáles debiesen ser las finalidades de la Historia, la Geografía y las Ciencias Sociales dentro del currículum. Esta asignatura debe contribuir a la democratización del país y a la construcción de una sociedad más justa y tolerante, así como a la educación de sujetos críticos, ciudadanos soberanos y conscientes que valoren la diversidad cultural como manifestación de riqueza social y que adscriban a un concepto de desarrollo que priorice la equidad social y la sostenibilidad ambiental.

Comparten también una similar concepción del conocimiento como una construcción en base a interpretaciones diversas y situadas, así como aproximaciones constructivistas y culturalistas respecto al aprendizaje. Se trata de ideologías curriculares explícitamente orientadas a la transformación cultural.

Es en sus postulados sobre la selección y organización de los contenidos, así como sobre las orientaciones para la enseñanza, donde estas ideologías se diferencian. Con todo, ambas consideran que la Historia y las Ciencias Sociales deberían integrar un solo sector de aprendizaje presente a lo largo de toda la escolaridad. Un sector de aprendizaje centrado en el estudio de la sociedad.

A pesar de todas estas coincidencias, es posible establecer una frontera entre la ideología crítico-disciplinar y la ideología crítico-pedagógica. Estas ideologías se diferencian por el énfasis en la dimensión disciplinar o pedagógica al momento de seleccionar y organizar los contenidos y orientar la enseñanza.

Para la ideología crítico-disciplinar, la selección de contenidos debe dar un lugar prioritario a las habilidades vinculadas a la búsqueda, análisis e interpretación de información, las que deben ser desarrolladas a través de una aproximación a los métodos de investigación de la historia, la geografía y las ciencias sociales. La secuencia debe articularse en torno a procesos históricos y sociales, considerando las dimensiones económica, espacial y cultural. En el estudio de la historia se le debe dar un lugar prioritario a la historia contemporánea y del presente, mientras que la geografía debe abordarse desde la perspectiva de la construcción social del espacio geográfico.

En esa lógica, las estrategias de enseñanza se deben orientar al desarrollo del pensamiento histórico y social por parte de los estudiantes. Lo anterior supone una constante exposición a una variedad de interpretaciones sobre la historia y la sociedad, al análisis crítico de información, así como a la introducción en los métodos de investigación de la historia y las ciencias sociales.

Por su parte, para la ideología crítico-pedagógica, los contenidos deben ser seleccionados y organizados de acuerdo a su relevancia para las experiencias y vivencias de los estudiantes, así como para la comprensión de su presente. En ese marco, el currículum debería organizarse temáticamente y contemplar tópicos como la memoria de la comunidad, la desigualdad y la estratificación social, la segregación socio-espacial y la 
degradación ambiental, así como también los conflictos económicos, sociales y étnicos vivos de la sociedad en distintas escalas.

Por su parte, la enseñanza debe considerar estrategias de aprendizaje social y colectivo, llevar los conflictos de la sociedad al aula y ofrecer oportunidades y herramientas para que los propios estudiantes sistematicen la realidad.

\section{DISCUSIÓN Y CONCLUSIONES}

Tal y como señala la teoría de referencia, la definición del currículum chileno de Historia, Geografía y Ciencias Sociales es el resultado de procesos profundamente políticos (Goodson, 2000), en los que un conjunto de actores e instituciones provenientes del campo político, intelectual y pedagógico (Bernstein, 1993) debaten y negocian las finalidades, los contenidos y las orientaciones para la enseñanza de la asignatura (Apple, 1996; Gvitz \& Palimedessi, 2005; Popkewitz, 1998).

En este artículo se identifican y describen las principales ideologías curriculares que promueven estos actores y agencias. Se ofrece así una visión esquemática de los principales intereses y discursos que participan de esta definición curricular. La intención detrás de este esquema es aportar a todos quienes les interesa o se ocupan de la enseñanza escolar de la historia y las ciencias sociales, una suerte de mapa cognitivo (Goodson, 2000) de esos actores y discursos. Como todo mapa, este es una representación de una realidad que siempre es más compleja. En este caso, en el ejercicio de identificar y distinguir los principales actores y discursos involucrados en la definición de la asignatura, se opacan matices, particularidades y excepciones. Es por eso que se explicitan las opciones teóricas y metodológicas en las que se sustenta esta interpretación, para hacer evidentes los supuestos y criterios sobre los que esta representación se construye.

Finalmente, al contar con una idea fundada de las ideologías curriculares que participan del debate y negociación de la asignatura, se abre la interrogante sobre el nivel de influencia de cada una de ellas en el currículum prescrito. Es decir, cuáles de estas ideologías han predominado, cuáles han sido consideradas y cuáles han sido eventualmente excluidas de las últimas versiones del currículum nacional. Respondiendo estas preguntas, se podría esbozar una suerte de trayectoria ideológica del currículum chileno de Historia, Geografía y Ciencias Sociales en la historia reciente. 
Estudios Pedagógicos XLIII, N ${ }^{\circ}$ 1: 157-169, 2017 IDEOLOGÍAS CURRICULARES EN EL DEBATE Y NEGOCIACIÓN DEL CURRÍCULUM CHILENO DE HISTORIA, GEOGRAFÍA Y CIENCIAS SOCIALES

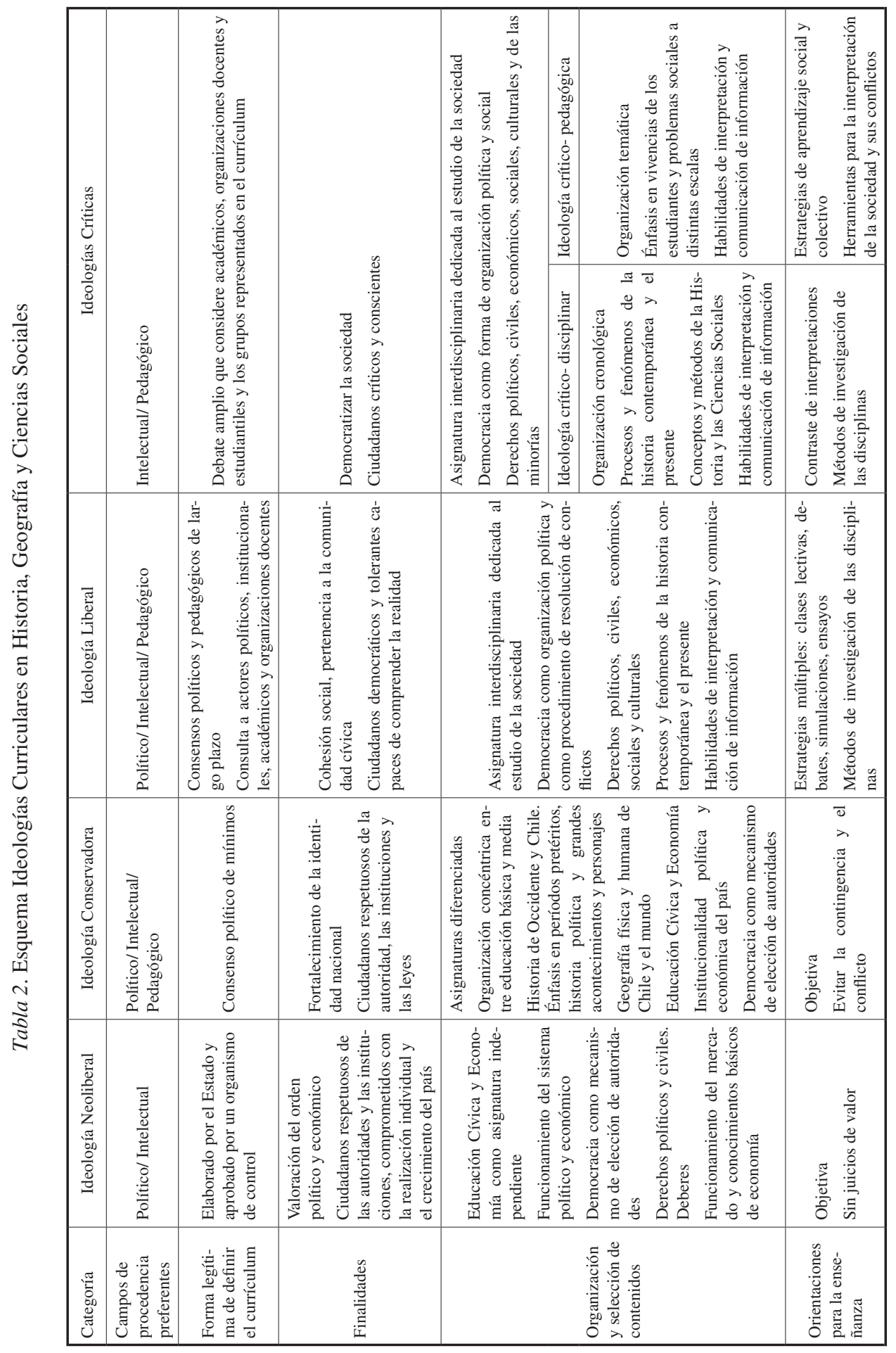


Estudios Pedagógicos XLIII, N 1: 157-169, 2017

IDEOLOGÍAS CURRICULARES EN EL DEBATE Y NEGOCIACIÓN DEL CURRÍCULUM CHILENO DE HISTORIA, GEOGRAFÍA Y CIENCIAS SOCIALES

\section{REFERENCIAS BIBLIOGRÁFICAS}

Apple, M. (1996). El conocimiento oficial. Buenos Aires: Paidós.

Barton, K., \& Levstik, L. (2004). Teaching history for the common good. New Jersey: Lawrence Erlbaum.

Benejam, P. (1997). Las finalidades de la educación social. En P. Benejam y J. Pagés (Eds.), Enseñar y aprender ciencias sociales, geografía e historia en la educación secundaria (pp. 33-51). Barcelona: Ice-Universidad de Barcelona/ Horsori.

Bernstein, B. (1993). La estructura del discurso pedagógico. Madrid: Morata.

Carrero, V., Soriano, R., \& Trinidad, A. (2012). Teoría fundamentada "Grounded Theory". El desarrollo de teoría desde la generación conceptual. Madrid: Centro de Investigaciones Sociológicas.

Carretero, M. (2007). Documentos de identidad. La construcción de la memoria histórica en el mundo global. Buenos Aires: Paidós.

Elliot, J. (1990). La investigación-acción en educación. Madrid: Morata.

Gazmuri, R. (2010). Propuesta de categorización de corrientes vigentes en la enseñanza escolar de la historia. Revista Íber, Didáctica de las ciencias sociales e historia, 63, 77-92.

Goodson, I. (2000). El cambio en el curriculum. Barcelona: Octaedro.

Goodson, I. (2003). Estudio del curriculum. Buenos Aires: Amorrortu.

Gvirtz, S., \& Palamidessi, M. (2005). El abc de la tarea docente: currículum y enseñanza. Buenos Aires: Aique Editores.

Joseph, B., Luster Barvman, S., Windschitl, M., \& Steward, N. (2000). Cultures of curriculum. New York: Lawrence Erlbaum Associates.

Kliebard, H. (2004). The struggle for the American curriculum, 1893-1958. New York: Routledge Falmer.

Levstik. L. (1996). Negotiating the history landscape. Theory and Research in Social Education, 24(4), 394-397.

Mayring, P. (2014). Qualitative content analysis: theoretical foundation, basic procedures and software solution. Klagenfurt: Gesis.

Ministerio de Educación. (1996). Objetivos Fundamentales y Contenidos Mínimos Obligatorios de la Educación Básica. Recuperado de http://ww2.educarchile.cl/UserFiles/P0001/File/ CurriculumBasica.pdf

Ministerio de Educación. (1998). Objetivos Fundamentales y Contenidos Mínimos Obligatorios de la Educación Media. Recuperado de http://www.agenciaeducacion.cl/wpcontent/uploads/2013/02/ MarcoCurriculardeEducMedia.pdf

Ministerio de Educación. (2004). Informe Comisión Formación Ciudadana. Recuperado de https:// tachistoriaudla.files.wordpress.com/2012/05/informe-formacic3b3n-ciudadana-en-chile.pdf

Ministerio de Educación. (2009). Objetivos Fundamentales y Contenidos Mínimos Obligatorios de la Educación Básica y Media. Recuperado de http://www.agenciaeducacion.cl/wp-content/ uploads/2013/02/Marco-Curricular-y-Actualizacion-2009-I-a-IV-Medio.pdf

Ministerio de Educación. (2012). Bases Curriculares 2012 Educación Básica. Recuperado de http:// ww2.educarchile.cl/UserFiles/P0001/File/CR_Articulos/introduccion.pdf

Ministerio de Educación. (2013). Nuevas Bases Curriculares 2013. Recuperado de http://lyd.org/ wp-content/uploads/2013/06/Mineduc.pdf

National Council for Social Studies. (2010). National Curriculum Standards for Social Studies: A Framework for Teaching, Learning, and Assessment. Maryland: Routledge.

Pagés, J. (1998). Models i tradicions curriculars en l'ensenyament de les ciències socials i de les humanitats: les finalitas i el valors com a opció ideològica. En P. Benejam y J. Pagés (Eds.), Psicopedagogia de les ciències socials $i$ de les humanitats (pp. 109-123). Barcelona: UOC.

Pagés, J. (2002). Aprender a enseñar historia y ciencias sociales: el currículo y la didáctica de las 
ciencias sociales. Pensamiento Educativo, 30(1), 255-269.

Popkewitz, T. (1998). Knowledge, power, and curriculum: Revisiting a TRSE argument. Theory and Research in Social Education, 26(1), 83-101. doi:10.1080/00933104.1998.10505835

Popuart, J. (1997). La recherche qualitative, enjeux épistémologiques et méthodologiques. Montreal: Gaëtan Morin.

Schiro, M. (2007). Curriculum theory: conflicting visions and enduring concerns. Thousand Oaks: Sage Publications.

Schubert, W. (1996). Perspectives on Four Curriculum Traditions. Educational Horizons, 74(4), 169176.

Strauss. A., \& J. Corbin. (2002). Bases de la investigación cualitativa: técnicas y procedimientos para desarrollar la teoría fundada. Antioquía: Universidad de Antioquía. 
